Marek A. Rostkowski

Pontificia Università Urbaniana

Città del Vaticano

\title{
La dimensione ecclesiale della vocazione missionaria dei laici nell'insegnamento post-conciliare
}

\section{The Ecclesiastical Dimension of the Missionary Vocation of the Laity in Post-Council Teaching}

\begin{abstract}
In the post-Council period, one can find different attempts at a synthesis of the declarations of the Council with regards to the theme of the apostolate of the laity. The authors recognizes that the faithful participate in the mission of the Church, with a variety of ministries, functions, and services for the common good and for the benefit of the entire body through being active members. The text maintains that Christian vocation and mission are inseparably united in the life of the faithful - one implies the other.
\end{abstract}

\section{Keywords}

Ecclesiology, missionary vocation, apostolate, laity, Church teaching.

Prima di affrontare la complessa gamma del campo, in cui il fedele laico come persona e come presenza di Chiesa è invitato, bisogna esporre che cosa intende il Concilio Vaticano II con il termine "apostolato"1. Come è sottolineato nel decreto Apostolicam actuositatem ${ }^{2}$, quando si parla di apostolato si vuole

\footnotetext{
${ }^{1}$ Per la bibliografia degli studi sui diversi aspetti della teologia del laicato, pubblicati nel periodo post-cociliare, cfr. A. Antón, Principios fundamentales para una teologia del Laicado en la Eclesiología del Vaticano II, "Gregorianum" 68 (Roma 1987) p. 103, 108-110; R. Goldie, Laici, laicato, laicità. Bilancio di trent'anni di bibliografia, Roma, 1986.
}

${ }^{2}$ Apostolicam actuositatem, 2. 


\section{The Person and the Challenges \\ 152

intendere quell'attività del singolo battezzato o del popolo di Dio che, essendo orientata alla diffusione del Regno, rende partecipi tutti gli uomini della salvezza operata da Gesù Cristo. Il Concilio definisce più volte l'apostolato come "vocazione", cioè una chiamata, una grazia, una fortuna, un dono, un onore che corrisponde all'essere invitati dal Signore a prendere parte alla sua stessa missione di salvezza. D'altra parte, vocazione è anche una risposta dell'uomo a Dio che chiama, è dunque un dovere, un impegno ed una responsabilità. In questo modo, l'apostolato si configura come dialogo tra il Salvatore ed il singolo laico nella comunione di tutta la Chiesa ${ }^{3}$. Dialogo, poi, che esige donazione e sacrificio perché ogni apostolato cristiano è "quasi una generazione", vale a dire, "infusione di un germe di vita".

La vocazione apostolica dei laici è, allo stesso momento, universale e personale perché tutti sono chiamati e nessuno è escluso: è la stessa chiamata alla Chiesa a condividere la missionarietà ed a portare il "glorioso peso" della sua missione salvifica ${ }^{5}$. Tuttavia, ognuno è chiamato per nome, ricevendo doni e carismi propri per il bene di tutti. È una vocazione interiore che «è radicata nello stesso essere ricevuto col battesimo, divenuto "creatura nuova" in Cristo e quindi partecipe del suo amore missionario per la gloria del Padre e per la salvezza dei fratelli» ${ }^{6}$. Così, il laico non deve attendere un mandato speciale dichiarato dalla gerarchia per impegnarsi apostolicamente, perché il primo ed ineliminabile mandato viene prepotentemente dai sacramenti nelle profondità dell'essere cristiano. L'orizzonte della vocazione e della missione dei laici è teologico e, pertanto, trinitariocristologico, ecclesiale ed antropologico?.

Il Catechismo della Chiesa Cattolica del 1992 parla della necessità dell'iniziativa dei cristiani laici, quando si tratta di scoprire e di ideare mezzi, per permeare dei bisogni della dottrina e della vita cristiana le realtà sociali, politiche ed economiche ${ }^{8}$. Il documento riporta le parole di papa Pio XII: "I fedeli laici si trovano sulla linea più avanzata della vita della Chiesa; grazie a loro la Chiesa è il principio vitale della società. Per questo essi soprattutto devono avere una

${ }^{3} \mathrm{Cfr}$. Apostolicam actuositatem, 33.

${ }^{4}$ U. Poletti, Missioni e cooperazione missionaria, Roma 1966, p. 32.

${ }^{5}$ Lumen gentium, 33. Cfr. M. Dhavamony, Lay Christians in the Mystery of Christ, in Lay missionaries in the Third Millennium = Missionnaires laïcs au troisième millénaire, ("Studia Missionalia"; 49), Roma 2000, p. 25.

${ }^{6}$ D. Tettamanzi, Laici, in: L. Rossi - A. Valsecchi (ed.), Dizionario enciclopedico di teologia morale, Roma 1976, p. 493.

${ }^{7}$ Cfr. P. Scabini, Vocazione e missione dei laici nei documenti conciliari e postconciliari, "Rivista di vita spirituale" 41 (Roma 1987), p. 381.

${ }^{8}$ Catechismo della Chiesa Cattolica, n. 899. 
coscienza sempre più chiara non soltanto di appartenere alla Chiesa, ma di essere la Chiesa, cioè la comunità dei fedeli sulla terra, sotto la guida dell'unico capo, il Papa, e dei vescovi in comunione con lui. Essi sono la Chiesa".

La missione dei fedeli li costituisce cooperatori e continuatori degli Apostoli per portare a compimento la loro opera. In questo modo, come la loro vita cristiana ed i carismi ricevuti, la loro missione deve esercitarsi in modo da assimilarsi all'azione e all'opera degli Apostoli. Per quel che riguarda l'oggetto e le norme dell'azione, una missione apostolica è conferita ai fedeli ed esiste solamente come una partecipazione, associazione e compimento della missione degli Apostoli ${ }^{10}$. "La vocazione dei laici all'apostolato si radica nei sacramenti che configurano i credenti a Gesù Cristo sacerdote, profeta e re, e li abilitano a condividerne nella Chiesa la missione di salvezza" ${ }^{\prime 1}$. Lumen gentium fa notare che i laici "compiono, nella Chiesa e nel mondo, la missione propria di tutto il popolo cristiano". È opportuno notare che l'espressione "nella Chiesa e nel mondo", riferita alla missione dei laici, non deve essere interpretata in maniera disgiuntiva, come se i christifideles avessero una missione nella Chiesa e un'altra missione nel mondo, oppure come se un'unica missione fosse da loro esercitata a volte nella Chiesa e a volte nel mondo. Contrariamente, i fedeli laici realizzando la loro missione nel mondo realizzano la loro missione nella Chiesa, perché la Chiesa vive nel mondo, è formata da uomini che sono nel mondo, non dovendo essere mondani ${ }^{12}$.

${ }^{9}$ Pio XII, Discorso del 20.02.1946, citato anche da Giovanni Paolo II nell'esortazione apostolica Christifideles laici, 9 .

${ }^{10}$ Cfr. Fil 4,3; Col 1,7; 4,12; 5,13; Rm 16, 3-4.6-7; 1 Cor 16,15; Giovanni Paolo II, La Chiesa missionaria (Udienza generale, (19.IV.1995), Insegnamenti di Giovanni Paolo II, Città del Vaticano, 1978-2006 (=IGPII), XVIII/1, 1009-1011; A. Scola, La missione della Chiesa all'alba del terzo millennio: discepoli e testimoni del Signore, in Pontificium Concilium pro Laicis, Il Congresso del laicato cattolico; Roma 2000 (Laici oggi), Città del Vaticano 2002, 89-93.

${ }^{11}$ Vocazione e missione dei laici nella Chiesa e nel mondo a vent'anni dal Concilio Vaticano II: Lineamenta, Città del Vaticano 1985, p. 25. Cfr. C. Colombo, Il laicato nella Chiesa, in Il laicato cattolico dei paesi di missione. Atti della seconda settimana di studi missionari, Milano, 4-8 settembre 1961, Milano 1962, p. 181; P.C. Landucci, Fondamenti dommatici dell'apostolato dei laici nella Chiesa, in: I laici nella Chiesa, (Iniziativa. Quaderni speciali; 2), Roma 1963, p. 24s.; A. Scola, La missione della Chiesa, p. 83; P. Coughlan, The Hour of the Laity. Their Expanding Role. Exploring "Christifideles laici" the Pope's key document on the laity, Philadelphia (PA) Newtown (NSW) 1989, p. 111s.; S. Fagan, Vocation and Mission of the Laity in the Church and the World. Twenty Years after the Second Vatican Council, "SEDOS Bulletin" 87 (Roma 1987), p. 19.

${ }^{12}$ Cfr. Giovanni Paolo II, Missione e missioni (Udienza generale, (03.V.1995), IGPII XVIII/1, p. 1181s.; F. Ocáriz, La partecipazione dei laici alla missione della Chiesa, "Annales theologici" 1 (Roma 1987), p. 10. 
La vocazione specifica del laico, evidenzia Y. Congar, cioè quella che corrisponde alla sua condizione in quanto tale, è di andare a Dio pur compiendo l'opera di questo mondo, costruendo la sostanza del mondo e della storia. Il laico deve vivere per Dio, ma senza essere dispensato dal fare l'opera del mondo, deve procurare la gloria di Dio e il regno di Cristo "in questo mondo e con l'opera di questo mondo: egli deve essere la Chiesa [...] operante, là dove i preti non lo sono, nella maniera in cui i sacerdoti non possono esserlo e, precisamente, nel temporale e nella storia, facendo l'opera del mondo e della storia"'l3.

Nel periodo post-conciliare si possono reperire le diverse prove di una sintesi delle dichiarazioni del Concilio riguardo al tema dell'apostolato dei laici. E. Niermann afferma che "il fatto che il laico assuma nella Chiesa il ruolo che gli compete, (...) dipende dalla formazione della coscienza interna alla Chiesa che è decisamente determinata dalla autocomprensione e dalla capacità di attuarsi in senso teologico della Chiesa" ${ }^{\prime 4}$. La vocazione cristiana è un impegno di partecipazione alla missione salvifica della Chiesa e tuttavia l'apostolato è per tutti, chierici e laici, sia dovere che diritto. Come rileva Niermann, i testi del Concilio, per designare questa attività laica, impiegano l'espressione tradizionale di "apostolato dei laici", in una accezione così lontana dal significato originale del termine che sono necessarie spiegazioni per non correre il rischio di restringere questa attività alla sola "collaborazione spirituale"15.

In una Chiesa ministeriale impegnata a manifestare e realizzare il piano di Dio nel mondo e nella storia (Ecclesia ministrans) ${ }^{16}$, esiste una missione comune a tutti i fedeli e unica dell'intero popolo di Dio, diversificata dal Signore in virtù del battesimo e dei doni del suo Spirito Santo. Tutti i fedeli partecipano alla missione della Chiesa, però con diversità di ministeri, di funzioni e servizi al bene comune e all'utilità dell'intero corpo, essendo loro membra attive ${ }^{17}$. Il cristiano come membro, è in relazione con gli altri membri dello stesso popolo di Dio, dell'unico corpo di Cristo, dello stesso tempio dello Spirito. Si può affermare

${ }^{13}$ Y. Congar, Per una teologia del laicato, Brescia 1967, p. 554. Cfr. R. Pellitero, La teologia del laicado en la obra de Yves Congar, Pamplona 1996, p. 224.

${ }^{14}$ E. Niermann, Laico, in: K. Rahner (ed.), Sacramentum mundi. Enciclopedia teologica, Brescia 1974-1977, IV, col. 655s.

${ }^{15}$ Ibid., col. 660s.

${ }^{16}$ Cfr. Ad gentes, 9; Giovanni Paolo II, Dare giusta autonomia ai laici in ciò che è di loro competenza senza ridurli a semplici collaboratori dei ministri ordinate (Ai Presuli della Conferenza Episcopale Brasiliana della Regione Nord 1 in: visita "ad limina", (30.V.1995), IGPII XVIII/1, p. 1604.

${ }^{17}$ Cfr. Apostolicam actuositatem, 2; P. Scabini, Apostolato, in: E. Ancilli (ed.), Dizionario di spiritualità dei laici, Milano 1981, p. 35; J. Aumann, The Role of the Laity, p. 159. 
che il discorso sulla missione laicale trova il suo fondamento ed il suo terreno di sviluppo nell'individuazione riguardante il posto del laico nel mondo e nella Chiesa $^{18}$.

L'altro aspetto dell'identità del fedele-laico viene dal fatto che egli è un appartenente al nuovo popolo di Dio e, in quanto tale, un membro vivo del Corpo mistico di Cristo che è la Chiesa ${ }^{19}$. I laici compiono la missione prima e principale della Chiesa "vivendo essi stessi per primi il mistero del Cristo, sia nella loro vita interiore personale di fede, di preghiera, di sacrificio e di amore, sia in modo pubblico partecipando alla attività ecclesiali di fede, di culto, di carità e di diaconia" ${ }^{20}$. Il Concilio Vaticano II presenta la Chiesa come mistero di comunione missionaria, come una "comunione organica", nella quale esistono diversità e complementarità di vocazioni, ministeri, servizi, carismi e responsabilità. I laici fanno parte della missione evangelizzatrice della Chiesa ed esercitano questa missione in diversi modi. Uno di questi è la testimonianza di vita o di esempio, e testimonianza della Parola, che si integrano perfettamente. Dalla fede all'azione, passando per la vita ed il suo valore di esempio, si manifesta in una identica confessione della fede.

Il dovere missionario è proprio di ciascuno e, in conseguenza, di tutti i fedeli, in virtù dei sacramenti dell'iniziazione cristiana, il battesimo, la cresima e l'eucaristia che li incorpora a Cristo come sue membra. Questo stretto obbligo missionario si realizza vivendo profondamente la specifica vita cristiana nel proprio ambiente, nello spirito cristiano tramite le preghiere, vocazioni ed aiuti ${ }^{21}$.

Il dovere dei laici all'apostolato non è una cosa nuova nella dottrina della Chiesa, essendo ormai numerosi i documenti pontifici in merito, a partire dal pontificato di Pio XI fino a quello di Paolo VI. Richiamando gli obblighi che i sacramenti di battesimo e di cresima impongono ad ogni cristiano, indicano,

${ }^{18}$ Cfr. C. Koser, Cooperazione dei laici con la gerarchia nell'apostolato, in: G. Baraúna (ed.), La Chiesa del Vaticano II. Studi e comenti intorno alla Costituzione dommatica Lumen Gentium, Firenze 1965, p. 998s.

19 "Infatti i credenti in Cristo, essendo stati rigenerati non di seme corruttibile, ma di uno incorruttibile, che è la parola del Dio vivo (cfr. 1 Pt 1,23), non dalla carne ma dall'acqua e dallo Spirito Santo (cfr. Gv 3,5-6), costituiscono "una stirpe eletta, un sacerdozio regale, una nazione santa, un popolo tratto in salvo..." Quello che un tempo non era neppure popolo, ora invece è popolo di Dio" (1 Pt 2,9-10)". (Lumen gentium, 9).

${ }^{20}$ Y. Congar, Laico, in: H. Fries (ed.), Dizionario teologico, Brescia 1966-1968, II, p. 138. Cfr. Idem, Apports, richesses et limites du Décret, in L'apostolat des laïcs. Décret Apostolicam actuositatem, (Unam Sanctam; 75), Paris 1970, pp. 171-181.

${ }^{21}$ Cfr. U. Poletti, Cooperazione tra le Chiese: implicazioni pastorali, in: E. Bartoletti, [ed al.], Chiesa locale e cooperazione tra le Chiese. Settimana di studi missionari, Assisi 10-15 settembre 1973, Bologna 1973, p. 128. 
come dovere verso Dio e verso il prossimo, l'apostolato anche per quel che riguarda i laici. Il laico è titolare della missione di tutta la Chiesa, così anch'egli è missionario e responsabile della edificazione della comunità cristiana ${ }^{22}$. In questo modo l'apostolato del laico viene specificato con l'espressione che egli deve "portare la Chiesa nel mondo ed il mondo nella Chiesa"23.

I laici non intervengono apostolicamente sulla base di un funzione, oppure di una missione ricevuta, ma sulla base di quello che hanno personalmente interiorizzato della vita cristiana. Così non è ex missione, ma ex spiritu ${ }^{24}$. Il dovere di esercitare l'apostolato non nasce così dalla chiamata della gerarchia, ma sgorga dal battesimo e dalla confermazione, e dalla carità che è il principio animatore di tutto apostolato. Essendo chiamati per mezzo dei sacramenti a partecipare, secondo la condizione loro propria, alla missione della Chiesa nel mondo, i laici hanno il diritto-dovere all'apostolato ${ }^{25}$. L'ulteriore chiamata della gerarchia può costituire la vocazione ad una particolare forma d'apostolato quale, ad esempio, quella dell'Azione Cattolica.

La missione del laico si fonda sulla consacrazione, il dare lo Spirito Santo nell'averlo ricevuto e nel riceverlo ogni istante. Come afferma Apostolicam actuositatem, la vocazione all'apostolato, similmente alla vocazione all'insieme delle attività ordinate a rendere partecipi tutti della redenzione operata da Gesù Cristo, si identifica con la vocazione cristiana ${ }^{26}$. Y. Congar afferma che la funzione apostolica è di tutta la Chiesa, ne fa parte organica e si distribuisce nelle sue membra nella misura delle mansioni e dei carismi dei singoli. "Al loro posto e secondo i loro talenti, i fedeli sono un unico soggetto con i loro sacerdoti nei vari

${ }^{22}$ Il concetto della communio christifidelium, concetto chiave della dottrina ecclesiologica del Vaticano II, è stato una delle linee direttrice nella sistemazione della nuova legislazione canonica, conclusa con la pubblicazione del nuovo Codice di Diritto Canonico (A. Antón, Fundamentos cristologicos, pp. 148-150).

${ }^{23}$ Cfr. M. Vergottini, La figura del laico nella letteratura teologica recente, in: A. Cargnel (ed.), Laicità e vocazione dei laici, Cinisello Balsamo 1987, p. 24; S.J. Emmanuel, Contemporary Catholic Thought on the Vocation and Mission of the Laity in the Church and in the World, (FABC Papers; 44), [Hong Kong] [1986], p. 31s.

${ }^{24}$ Cfr. Y. Congar, Pour une théologie du laïcat, „Études” (Paris 1948) Tome 256, p. 203.

${ }^{25}$ Cfr. J. Bruls, La vocation du laïc missionnaire, in Secrétariat International du Laïcat Missionnaire, Semaine d'études du S.I.L.M. Würzburg, du 2 au 8 janvier 1954, Milan 1954, p. 13.

${ }^{26} \mathrm{Cfr}$. Apostolicam actuositatem, 2 e 3 . Vedi: J. Ratzinger, La mission d'après les autres textes conciliaires, in L'activité missionnaire de l'Église. Décret Ad gentes, (Unam Sanctam; 67), Paris 1967, p. 133s.; B. Forte, Laicato e laicità. Saggi ecclesiologici, Genova 1986, p. 47s.; J. Esquerda Bifet, Iglesias hermanas en la misión. Cooperación entre Iglesias particulares y distribución de efectivos apostólicos, Madrid 1981, p. 13. A. Seumois afferma che la carità teologale verso il prossimo, secondo Gv 15,12, è il fondamento morale costitutivo di tutta la vitalità ecclesiale, del suo ministero e dell'opera missionaria in particolare (Teologia missionaria, p. 286). 
organismi della Chiesa, una sola persona (morale), che ha, come tale, una stessa responsabilità missionaria e personale" ${ }^{27}$. Più specificamente l'autore sviluppa le due direzioni in cui si attua l'apostolato verso il mondo: evangelizzazione e cristianizzazione ("civilizzazione"): "Il primo compito è di evangelizzazione o di pura testimonianza della fede e della carità di Cristo; esso mira a convertire gli uomini al Vangelo. Il secondo consiste nell'influenzare l'ordine temporale, cioè tutta l'opera della Città terrestre degli uomini [...]"28.

In ambedue gli oggetti della missione della Chiesa, cioè l'evangelizzazione e la civilizzazione, sono impegnati sia laici che chierici, però in modo diverso. Secondo Y. Congar l'opera di cristianizzazione o animazione delle realtà temporali sembra più propria dei laici.

Il Papa Wojtyła sottolinea: "Grazie a questa diversità e complementarietà ogni fedele laico si trova in relazione con tutto il corpo e ad esso offre il proprio contributo" 29 , cioè non contrapposizioni e divisioni, ma reciprocità e coordinamento. Così il Vaticano II supera definitivamente una unilaterale identificazione della Chiesa con la gerarchia, per lungo tempo dominante nell'ecclesiologia, ed apre la strada alla riscoperta della vocazione laicale ${ }^{30}$. Oltre alla condizione battesimale dei laici, come esigenza di partecipazione attiva alla missione della Chiesa, l'esortazione Christifideles laici menziona anche la loro specifica vocazione e fa una importante precisazione al riguardo: "I vari ministeri, uffici e funzioni che i fedeli laici possono legittimamente svolgere nella liturgia, nella trasmissione della fede e nelle strutture pastorali della Chiesa, dovranno essere esercitati in conformità alla loro specifica vocazione laicale, diversa da quella dei sacri ministri”31.

${ }^{27}$ Y. Congar, Laico, p. 140.

${ }^{28}$ Y. Congar, Sacerdozio e laicato di fronte ai loro compiti di evangelizzazione e di civiltà, Brescia 1966, p. 238. Cfr. J.-É. Champagne, Le laïcat missionnaire, in Le laïcat et les missions. Rapport de la quatrième semaine d'Études missionnaires du Canada, tenue à Montreal, du 16 au 22 octobre 1950, Ottawa 1951, p. 85; M. Candia, Missionswesen des Laienstandes, Milano 1952, p. $9 \mathrm{~s}$.

${ }^{29}$ Christifideles laici, 20.

${ }^{30}$ Cfr. S. Ryłko, Il Concilio Vaticano II, pietra miliare nel camino del laicato cattolico, in Pontificium Concilium pro Laicis, Il Congresso del laicato cattolico; Roma 2000, (Laici oggi), Città del Vaticano 2002, pp. 120-122.

${ }^{31}$ Christifideles laici, 23. Cfr. Giovanni Paolo II, Ob diem ad Missiones fovendas, (11. VI.2000), AAS 92 (2000) p. 686; E. Pironio, Vocazione e missione dei laici alla luce del Sinodo 1987, in: V. Liberti, (ed.), I laici nel popolo di Dio: esegesi biblica, Roma 1990, p. 14s.; J.R. Villar, La partecipación de los cristianos laicos en la misión de la Iglesia, "Scripta theologica" 33 (Pamplona 2001), pp. 659-661. 


\section{The Person and the Challenges \\ 158 Volume 2 (2012) Number 2}

Nella vita del fedele cristiano vocazione e missione sono intrinsecamente unite - l'una implica l'altra. L'identità del laico si costruisce non solo mediante un'analisi teologica del suo essere, ma in modo particolare mediante un concreto impegno apostolico-missionario. Cristo il suo mandato missionario l'ha affidato a tutta la Chiesa, perciò tutti i cristiani sono chiamati all'apostolato. Il Concilio Vaticano II afferma nella Lumen gentium: "I laici [...] sono chiamati chiunque essi siano, a contribuire come membra vive, [...] all'incremento della Chiesa e alla sua santificazione permanente. L'apostolato dei laici è quindi partecipazione alla missione salvifica stessa della Chiesa; a questo apostolato sono tutti destinati dal Signore stesso per mezzo del battesimo e della confermazione. Dai sacramenti poi, e specialmente dalla sacra eucaristia, viene comunicata e alimentata quella carità verso Dio e gli uomini che è l'anima di tutto l'apostolato. Ma i laici sono soprattutto chiamati a rendere presente e operosa la Chiesa in quei luoghi e in quelle circostanze in cui essa non può diventare sale della terra se non per loro mezzo. Così ogni laico, in virtù dei doni che gli sono stati fatti, è testimone $\mathrm{e}$ insieme vivo strumento della stessa missione della Chiesa « secondo la misura del dono del Cristo » (Ef 4,7)"32.

Una simile affermazione si trova anche nel primo articolo del decreto sull'apostolato dei laici Apostolicam actuositatem: "L'apostolato dei laici, infatti, derivando dalla loro stessa vocazione cristiana, non può mai venir meno nella Chiesa. [...] I nostri tempi poi non richiedono minore zelo da parte dei laici; anzi le circostanze odierne richiedono assolutamente che il loro apostolato sia più intenso e più esteso" 33 .

Questo documento conciliare è in diretta relazione con il capitolo IV della Lumen gentium, di cui sviluppa i principi per fornire una panorama d'insieme dell'apostolato dei laici. In questo modo, l'intima connessione del decreto con la costituzione sulla Chiesa si presenta come il suo naturale sviluppo e continuazione. La vocazione dei laici all'apostolato viene presentata come costitutiva del loro essere cristiani e membri della Chiesa. Come si sottolinea nei Lineamenta per VII Assemblea Generale Ordinaria del Sinodo dei Vescovi, "l'apostolato dei laici, derivato dall'unica missione della Chiesa e ordinato ad edificare l'unico Corpo di Cristo, esige di armonizzarsi con tutti gli altri ministeri e servizi presenti e operanti nel Popolo di Dio. È la «comune partecipazione» alla missione ecclesiale che insieme abilita ed impegna a crescere continuamente verso una matura

${ }^{32}$ Lumen gentium, 33. Cfr. Catechismo della Chiesa Cattolica, n. 900; A. Antón, Principios fundamentales, p. 141s.

${ }^{33}$ Apostolicam actuositatem, 1. 
«corresponsabilità ecclesiale», dalla quale soltanto può svilupparsi un'azione pastorale organica d'insieme" ${ }^{\prime 3}$.

Nessun ambiente o attività umana, evidenzia il documento successivo del Sinodo, Instrumentum laboris, può essere trascurato dalla presenza cristiana. In altre parole, si tratta di evangelizzare in modo capillare tutte le forme dell'esperienza umana, non dimenticando alcune aeree nelle quali la testimonianza dei laici assume un'importanza prioritaria. Il Papa Wojtyła sottolinea già all'inizio della Christifideles laici che i laici devono ripensare il loro ruolo nell'apostolato, nell'ottica dell'urgenza missionaria: "la Chiesa ha maturato una più viva coscienza della sua natura missionaria ed ha riascoltato la voce del suo Signore che la manda nel mondo come «sacramento universale di salvezza». Andate anche voi. La chiamata non riguarda soltanto i Pastori, i sacerdoti, i religiosi e le religiose, ma si estende a tutti: anche i fedeli laici sono personalmente chiamati dal Signore, dal quale ricevono una missione per la Chiesa e per il mondo" 35 .

Per quanto sia il ruolo dei fedeli laici in campo missionario, il decreto sull'attività missionaria della Chiesa mette in evidenza la "missionarietà" come caratteristica che appartiene ad ogni battezzato perché "tutti i fedeli, quali membra del Cristo vivente, a cui sono stati incorporati ed assimilati mediante il battesimo, la cresima e l'eucaristia, hanno lo stretto obbligo di cooperare all'espansione e alla dilatazione del suo corpo, sì da portarlo il più presto possibile alla sua pienezza"36.

Questo universale dovere missionario non deriva dal mandato della gerarchia, ma dall'unione con Cristo che spinge i fedeli all'apostolato. Ogni laico deve operare nella realtà temporale riunendo in sintesi i suoi doveri terreni, la sua ispirazione evangelica che lo fa evangelizzatore del mondo. Portare la Buona

\footnotetext{
${ }^{34}$ Lineamenta, 33. Cfr. Giovanni Paolo II, Unità nell'annuncio e responsabile proclamazione della verità: l'evangelizzazione ci spinge con forza verso questa duplice via (L'incontro con $i$ sacerdoti, $i$ religiosi e $i$ rappresentanti di associazioni e movimenti ecclesiali, Mantova, 22.VI.1991), IGPII XIV/1, p. 1731; Idem, Relazioni dei Presbiteri con gli altri fedeli (Udienza generale, 22.IX.1993),), IGPII XVI/2, p. 826s.

${ }^{35}$ Christifideles laici, 2.

${ }^{36}$ Ad gentes, 36. Giovanni Paolo II ribadisce nell'enciclica Redemptoris missio, quando, parlando del carattere missionario di tutto il popolo di Dio e, in modo particolare, dei laici, ricorda che "la necessità che tutti i fedeli condividano tale responsabilità non è solo questione di efficacia apostolica, ma è un dovere-diritto fondato sulla dignità battesimale per cui "i fedeli partecipano, per la loro parte, al triplice ufficio - sacerdotale profetico e regale di Gesù Cristo". (Redemptoris missio, 71). Cfr. Idem, Dal Vaticano II uno spazio vasto e aperto per i laici nella dimensione missionaria (Sinodo 1987: Vocazione e missione dei laici nella Chiesa e nel mondo - Regina coeli, 17.V.1987), IGPII X/2, p. 1711.
} 
Novella di Cristo appartiene alla missione di tutta la Chiesa, condotta dallo Spirito Santo, missione che è di tutti i membri della Chiesa. I fedeli laici, i christifideles, vivendo nella fede in Cristo, hanno ricevuto la specifica vocazione di salvaguardare questa testimonianza e questo servizio evangelico dell'uomo in tutti gli aspetti della vita personale e sociale ${ }^{37}$. Nel Ad gentes leggiamo: "La Chiesa non si può considerare realmente fondata, non vive in maniera piena, non è segno perfetto della presenza di Cristo tra gli uomini, se alla gerarchia non si affianca e collabora un laicato autentico. Non può infatti il Vangelo penetrare ben addentro nella mentalità, nel costume, nell'attività di un popolo, se manca la presenza dinamica dei laici. Perciò, fin dal periodo di fondazione di una Chiesa, bisogna dedicare ogni cura alla formazione di un maturo laicato cristiano" 38 .

La missione propriamente detta è uno dei campi più specifici per la testimonianza dei laici. Non potendo celebrare l'eucaristia, che è il sacramento del corpo di Cristo, essi sono incapaci di fondare pienamente la Chiesa e di farne il corpo di Cristo. Però possono iniziarla, suscitarla, dal momento che, prima di essere una comunità sacramentale eucaristica, la Chiesa vive come una comunità catecumenale, caratterizzata dalla fede e dalla preghiera ${ }^{39}$.

Il decreto Ad gentes invita i laici a cooperare con i pastori della Chiesa nel campo importante e vasto della pastorale missionaria, specialmente se, chiamati da Dio, vengono destinati dai vescovi all'impegno nell'opera evangelizzatrice, come testimoni e vivi strumenti alla sua missione salvifica ${ }^{40}$. Il nuovo Codice di Diritto Canonico ricorda che "nello svolgimento dell'opera missionaria siano assunti i catechisti, cioè fedeli laici debitamente istruiti ed eminenti per vita cristiana, perché, sotto la guida del missionario, si dedichino a proporre la dottrina evangelica e ad organizzare gli esercizi liturgici e le opere di carità’"41.

Un percorso sommario sulla problematica ecclesiologica della vocazione missionaria dei laici permette di rilevare la seguente conclusione: Nel periodo post-conciliare si possono trovare le diverse prove di una sintesi delle dichiarazioni del Concilio riguardo al tema dell'apostolato dei laici. Tutti gli autori riconoscono che i fedeli partecipano alla missione della Chiesa, però con diversità di ministeri,

${ }^{37}$ Cfr. Vescovi francesi, L'apostolat des laïcs, "Documentation catholique” 81 (Paris 1999), p. 169.

${ }^{38}$ Ad gentes, 21.

${ }^{39}$ Nella storia della Chiesa si ricorda che spesso gli inizi della sua esistenza e poi la sopravvivenza in tempo di persecuzione furono proprio grazie dei laici. Cfr. G. Philips, La Chiesa e il suo mistero nel Concilio Vaticano II. Storia, testo e commento della Costituzione "Lumen Gentium”, Milano 1969, II, p. 31.

${ }^{40} \mathrm{Cfr}$. Ad gentes, 41.

${ }^{41}$ Can. $785 \$ 1$. 
di funzioni e di servizi per il bene comune e per l'utilità dell'intero corpo, essendo loro membra attive. Sostengono che nella vita del fedele cristiano vocazione e missione sono inseparabilmente unite - l'una implica l'altra.

\section{Bibliography}

Colombo C., Il laicato nella Chiesa, in Il laicato cattolico dei paesi di missione. Atti della seconda settimana di studi missionari, Milano, 4-8 settembre 1961, Milano 1962, pp. 179-187.

Congar Y., Pour une théologie du laïcat, Études (Paris 1948) Tome 256, pp. 42-54; 194 218.

Congar Y., Sacerdozio e laicato di fronte ai loro compiti di evangelizzazione e di civiltà, Brescia 1966.

Congar Y., Per una teologia del laicato, Brescia 1967.

Coughlan P., The Hour of the Laity. Their Expanding Role. Exploring "Christifideles laici” the Pope's Key Document on the Laity, Philadelphia (PA) - Newtown (NSW) 1989.

Forte B., Laicato e laicità. Saggi ecclesiologici, Genova 1986.

Goldie R., Laici, laicato, laicità. Bilancio di trent'anni di bibliografia, Roma, 1986.

Koser C., Cooperazione dei laici con la gerarchia nell'apostolato, in: G. Baraúna (ed.), La Chiesa del Vaticano II. Studi e comenti intorno alla Costituzione dommatica “Lumen Gentium”, Firenze 1965, pp. 994-1011.

Landucci P.C., Fondamenti dommatici dell'apostolato dei laici nella Chiesa, in I laici nella Chiesa, (Iniziativa. Quaderni speciali; 2), Roma 1963, pp. 15-29.

Pironio E., Vocazione e missione dei laici alla luce del Sinodo 1987, in: V. Liberti (ed.), I laici nel popolo di Dio: esegesi biblica, Roma 1990, pp. 1-18.

Poletti U., Cooperazione tra le Chiese: implicazioni pastorali, in: E. Bartoletti, [ed al.], Chiesa locale e cooperazione tra le Chiese. Settimana di studi missionari, Assisi 1015 settembre 1973, Bologna 1973, pp. 127-149.

Ratzinger J., La mission d'après les autres textes conciliaires, in: L'activité missionnaire de l'Église. Décret “Ad gentes” (Unam Sanctam; 67), Paris 1967, pp. 121-147.

Ryłko S., Il Concilio Vaticano II, pietra miliare nel camino del laicato cattolico, in Pontificium Concilium pro Laicis, Il Congresso del laicato cattolico; Roma 2000, (Laici oggi), Città del Vaticano 2002, pp. 115-139.

Vergottini M., La figura del laico nella letteratura teologica recente, in: A. Cargnel (ed.), Laicità e vocazione dei laici, Cinisello Balsamo 1987, pp. 15-42. 
